\title{
Introdução à Robótica e Estímulo à Lógica de Programação no Ensino Básico Utilizando o Kit Educativo LEGO® Mindstorms
}

\author{
Ygor Q. de Aguiar ${ }^{1,2}$, Braian K. Maciel ${ }^{1,2}$, Sandro D. G. Mattos ${ }^{1,2}$, Luciane B. \\ Soares $^{1,2}$, Vinícius M. de Oliveira ${ }^{1,2}$. \\ Centro de Ciências Computacionais - C3 \\ ${ }^{2}$ Programa de Educação Tutorial Ciências Computacionais - PET-C3 \\ Universidade Federal do Rio Grande (FURG) - Rio Grande, RS - Brazil \\ \{ygorqa, braian.konzgen, sandro.gaubert, lucianebaldassari, \\ vinicius.oliveira\}@furg.br
}

\begin{abstract}
This article reports the pedagogical experience and the impact of the completion of the extension project "Lego in Schools". In partnership with elementary schools, the work has the aim to stimulate and teach programming logic in a different and inductively. It is believed that the teaching of basic engineering and computing, by solving problems with robotics and programming, contributes to child training and arouses the interest for science and technology. For this, we used the educational LEGO® Mindstorms kit, which has an easy learning software and is used for various educational purposes.
\end{abstract}

Resumo: Este artigo relata a experiência pedagógica e o impacto da realização do projeto de extensão "Lego nas Escolas". Em parceria com escolas de ensino fundamental, o trabalho possui o intuito de estimular e ensinar a lógica de programação de uma forma diferente e indutiva. Acredita-se que o ensino da base da engenharia e da computação, através da resolução de problemas com a robótica e programação, contribui com a formação infantil e desperta o interesse para as áreas da ciência e tecnologia. Para isto, foi utilizado o Kit educativo LEGO® Mindstorms, que possui um software de fácil aprendizagem e é utilizado para diversos fins educacionais.

\section{INTRODUÇÃO}

A sociedade está cada vez mais diretamente dependente do emprego das ferramentas tecnológicas para realização de suas tarefas diárias, seja no meio do trabalho ou simplesmente como forma de lazer. No entanto, esse aumento relativo no uso da tecnologia não reflete um crescimento no interesse das recentes gerações no aprendizado em ciências e tecnologia (MITCHELL, 2000).

Portanto, medidas para combater essa tendente característica já foram tomadas em alguns países onde disciplinas de lógica de programação e introdução a robótica foram incluídas no projeto pedagógico do ensino fundamental e médio (CARBONE, 2014).

A importância da inclusão dessas disciplinas, que além de favorecer o interesse dos alunos para as ciências exatas e tecnológicas, contribui para o aprendizado de todas as disciplinas de uma maneira geral. Isso deve-se à característica interdisciplinar do ensino de engenharia, que possibilita a conexão entre os conteúdos aprendidos nas diversas disciplinas da 
grade curricular para a resolução de problemas, através da lógica e do conhecimento cientifico (ROGERS \& PORTSMORE, 2004).

Considerando a importância da inclusão de disciplinas de engenharia no ensino básico para estimular a lógica na identificação, formulação e resolução de problemas, desenvolve-se um projeto de extensão denominado "Estímulo à lógica de programação através do uso do kit educativo LEGO ${ }^{\circledR}$ Mindstorms", cujo público-alvo são alunos advindos do Ensino Fundamental das escolas públicas.

O objetivo principal do projeto de extensão desenvolvido é de estimular o raciocínio lógico e instigar uma nova maneira de pensar, apropriando-se, de uma maneira simples e interativa, de conhecimentos de lógica de programação e robótica. Como mencionado anteriormente, a escolha do uso da robótica como objeto de ensino se deu pelo fato de ser uma temática que engloba aspectos da física e da matemática, que, por sua vez, são matérias que constam no conteúdo programático das escolas de Ensino Fundamental e Médio. O ensino da lógica de programação é aplicado aos alunos visando introduzir o conhecimento, mesmo que de forma sucinta, de como solucionar problemas através de uma sequência de passos definida, fazendo uso de um pensamento mais voltado à lógica matemática.

\section{METODOLOGIA}

Tendo em vista tornar o ensino da lógica de programação o mais simples e interessante possível, optou-se por utilizar um kit educacional da LEGO ${ }^{\circledR}$, o Mindstorms. Este kit possui uma interface projetada para ser dinâmica e atrativa, utiliza blocos de comando coloridos que se conectam uns aos outros pelo simples arrasto do mouse na tela do computador, o que possibilita a formação de sequências de ações que em seguida são enviadas ao robô (DE OLIVEIRA et al., 2013). Diversas peças integram o kit, isso permite ao usuário montar o modelo de robô que desejar, o que estimula a criatividade e é uma característica dos produtos da LEGO®.

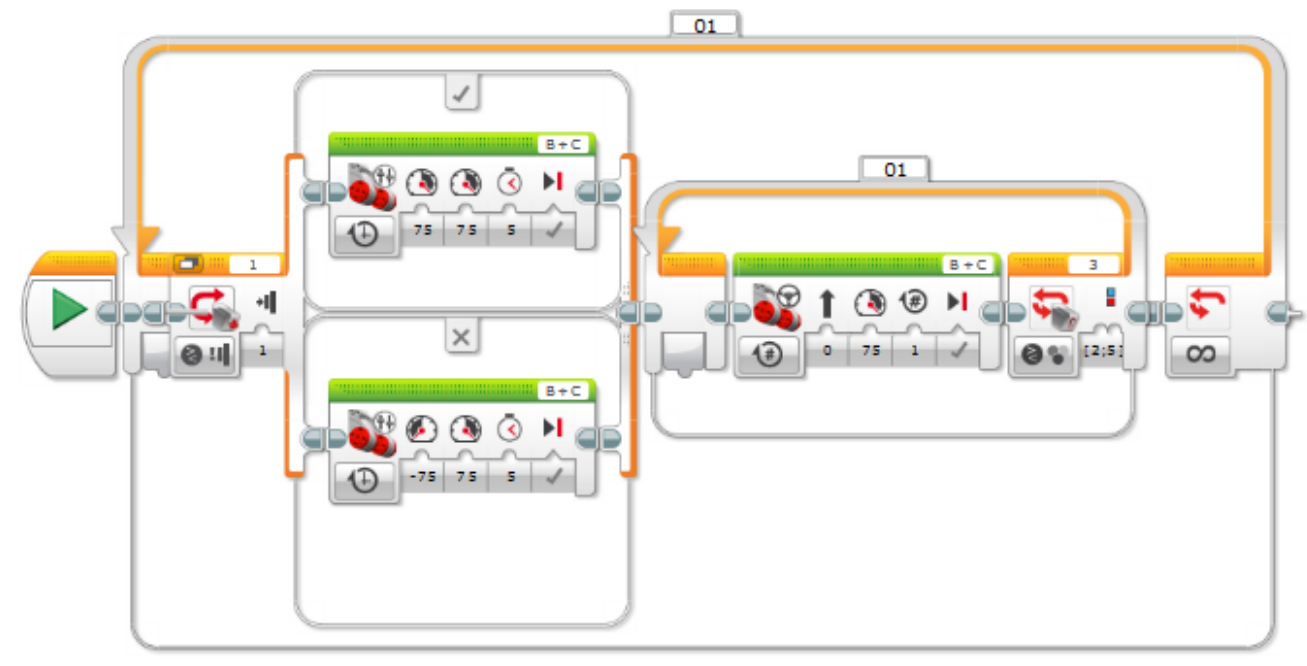

Figura 1. Blocos de comandos para execução de uma atividade.

O kit utilizado dispõe de diversos componentes para a montagem dos robôs como itens para a estrutura do robô e diversos sensores e atuadores. Essa característica permite ao usuário montar uma grande gama de robôs estimulando a criatividade na resolução de problemas.

Em um primeiro momento foi necessária a realização da capacitação de todos no projeto. A capacitação se justificou para que pudéssemos partir de um mesmo ponto através do nivelamento dos membros do projeto. A capacitação foi realizada com o auxílio de bolsistas do grupo de robôs de LEGO® da Universidade. Durante a capacitação, foram desenvolvidos códigos através do software do kit educativo e também foram realizadas montagens de diversos modelos de robôs. 
Após a capacitação dos membros do projeto o próximo passo foi o contato com as escolas públicas para fazer a divulgação do projeto e iniciar as atividades de ensino. As atividades tiveram duração de aproximadamente um mês contaram com um encontro semanal de quatro horas, totalizando 16 horas. Foram convidadas turmas de oitava série ( $9^{\circ}$ ano) e estas divididas em grupos, os quais foram instruídos no desenvolvimento das atividades propostas. Dentre estas atividades estão:

a. a familiarização com o ambiente de programação do Mindstorms;

b. a apresentação dos comandos do software;

c. a apresentação dos componentes e sensores dos robôs;

d. a montagem dos robôs;

e. a criação de algoritmos para solução dos problemas e desafios propostos.

Os alunos contaram com o auxílio de um material didático desenvolvido especialmente para o projeto, além da supervisão no decorrer de cada tarefa. O material didático desenvolvido, Figura 2, é uma apostila de 35 páginas contendo uma introdução à lógica de programação, o emprego de fluxogramas, e a explicação do uso dos componentes do kit e software educativo, assim como exercícios e atividades de desenvolvimento.

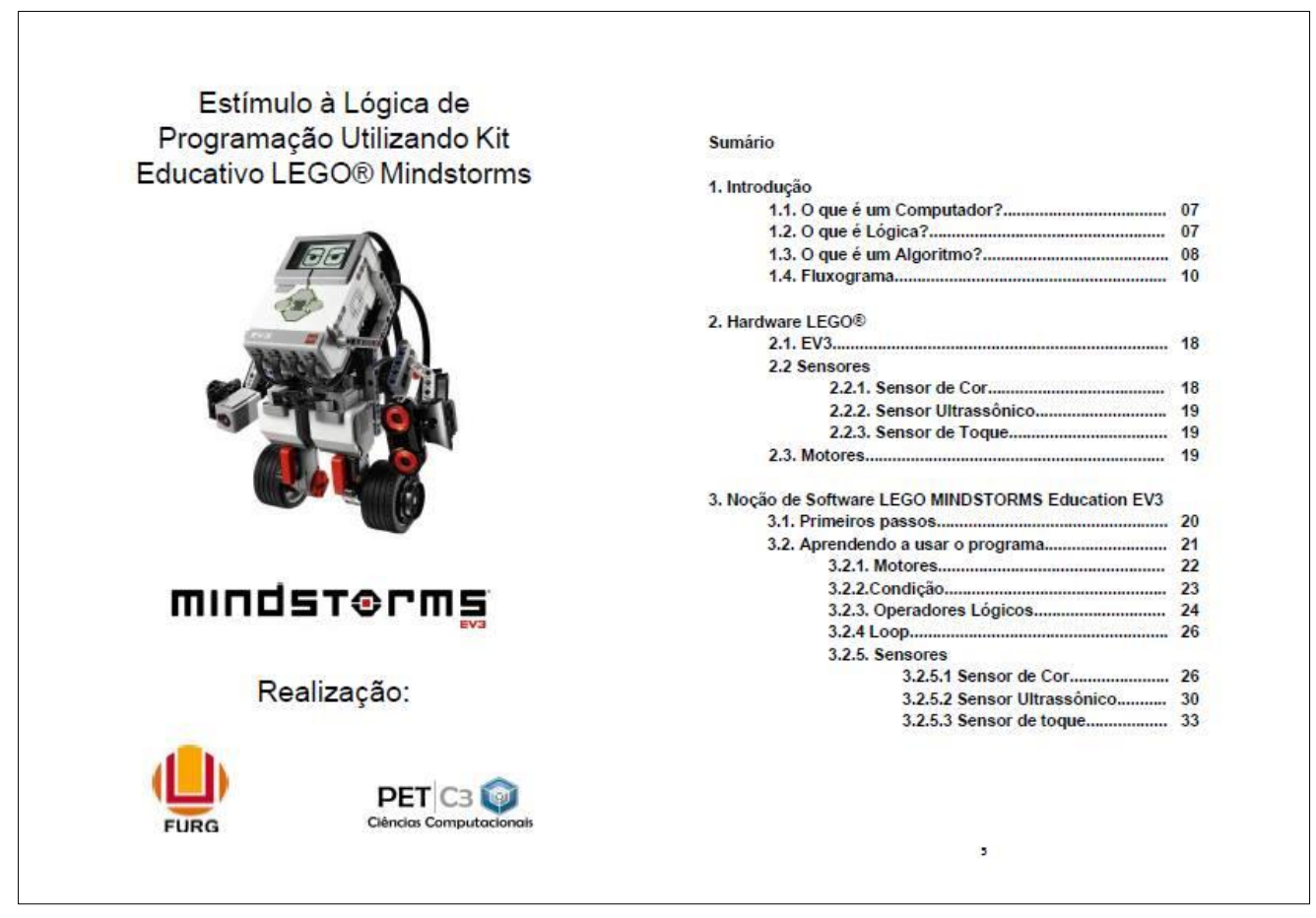

Figura 2. Apostila desenvolvida para o curso

Ao término das aulas propusemos um desafio final em que os grupos envolvidos no projeto se enfrentaram em uma competição que englobou todo o conteúdo apresentado. Os estudantes precisavam utilizar dois tipos de sensores (sensor de cor e de distância) para formular a lógica de solução do problema dado. O desafio consistia em fazer com que o robô percorresse um circuito proposto (Figura 3 ) utilizando os sensores. 
CBIE-LACLO 2015

Anais dos Workshops do IV Congresso Brasileiro de Informática na Educação (CBIE 2015)

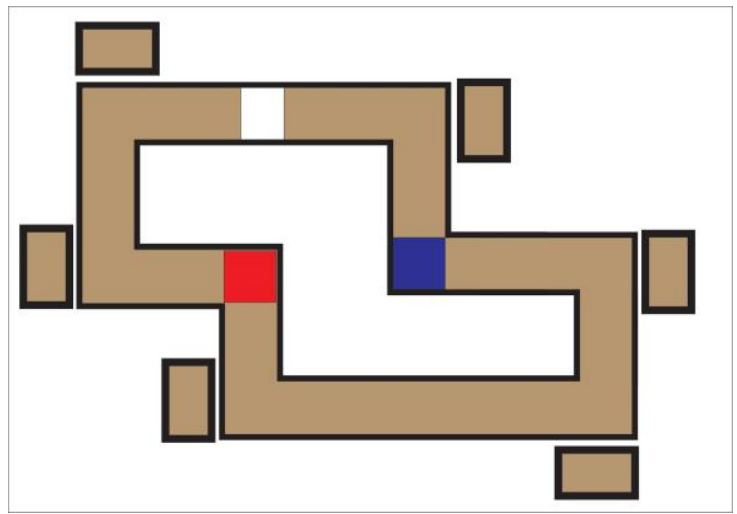

Figura 3. Circuito proposto para o Desafio Final

As únicas informações que foram fornecidas para a solução do problema foram as seguintes:

- Sensor de cor laterais, utilize os dois sensores para o robô se manter dentro do circuito;

- Sensor de cor central, quando identificar a cor azul ou vermelha o robô deve dobrar a esquerda;

- Sensor de distância, quando identificar uma distância de $10 \mathrm{~cm}$ o robô deve dobrar para a direita;

- O inicio e o fim do circuito é marcado na faixa branca.

No início do curso, aplicamos um questionário que teve como intuito traçar o perfil do aluno, o questionário é composto por perguntas, como por exemplo, "Com que frequência você usa o computador?", "Você gosta de desafios matemáticos/lógica?", "Você já teve algum contato com programação de computadores? Se sim, onde?Quanto tempo?", entre outras.

Com o intuito de analisar o resultado obtido após a realização do projeto, foi adotado a aplicação de dois testes de lógica, um no início do curso (Figura 4), para medir o nível de lógica dos alunos, e outro no final do curso (Figura 5), com intenção de comparar com o primeiro teste, para identificar a contribuição da atividade proposta para o desenvolvimento do pensamento lógico no ensino básico. As questões dos dois testes são diferentes, mas ambos se encontravam no mesmo nível de dificuldade.

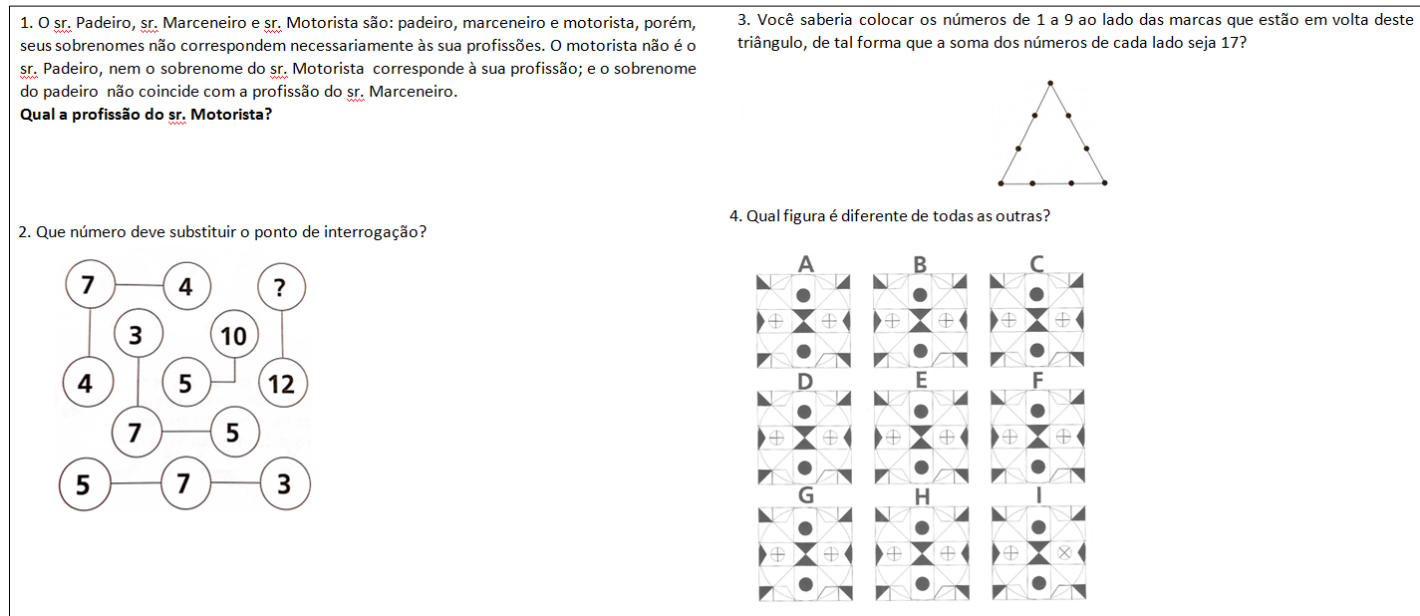

Figura 4. Primeiro teste de lógica aplicado 

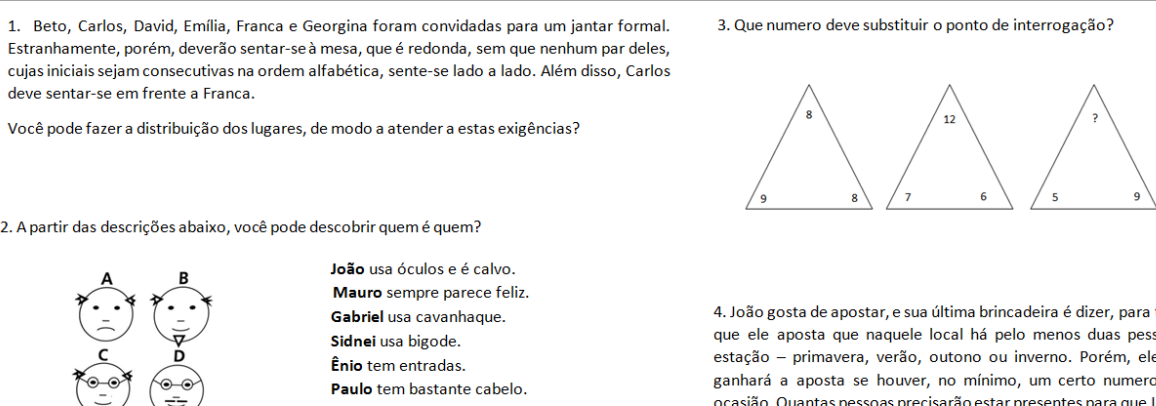

Figura 5. Segundo teste de lógica aplicado

\section{RESULTADOS E DISCUSSÕES}

Quanto ao software, os alunos o receberam muito bem, pois o mesmo possui uma interface muito simples. Uma vez que se aprende a usá-lo, se torna simples passar alguma tarefa para o robô, o que com o tempo, foi sendo aperfeiçoado pelos alunos. Já em relação ao desempenho nos desafios propostos, os alunos no início do primeiro problema tinham um certo receio, sem saber se iriam conseguir fazer com que o robô desempenhasse a tarefa corretamente, porém com o passar das aulas e dos desafios sendo executados, sua confiança com o software foi aumentando, conseguindo assim, realizar as tarefas mais rapidamente devido ao estímulo de seu raciocínio lógico. Notou-se nitidamente a evolução dos alunos do início do projeto ao seu fim.

Verificando as respostas dos questionários, identificamos que a faixa etária dos alunos, se encontrou entre 14 e 15 anos. Concluímos também que grande parte dos alunos usa diariamente o computador; dois terços responderam, que gostam de desafios lógicos; e a maioria nunca teve contato, com algum tipo de programação de computadores.

Tabela 1. Informações Gerais do Projeto

\begin{tabular}{|l|l|l|}
\hline & Teste de Lógica 1 & Teste de Lógica 2 \\
\hline Média & 5.6 & 5.3 \\
\hline Desvio Padrão & 3.9 & 2.8 \\
\hline Tempo médio Total & 17 min e $14 \mathrm{seg}$ & 14 min e $06 \mathrm{seg}$ \\
\hline
\end{tabular}

Segundo a Tabela 1, a média total de acertos no primeiro teste de lógica aplicado foi de 5.6, com desvio padrão de 3.9, e média de tempo de 17 minutos e 14 segundos para realizar todo o teste. A média total de acertos no segundo teste foi 5.3, baixando 3 décimos, porém o desvio padrão reduziu para 2.8 , uma diferença de $1.1 \mathrm{em}$ relação ao primeiro teste. Com a redução do desvio padrão, podemos afimar que a dispersão na média diminuiu, indicando que a desenvoltura dos alunos tenderam a estar próximos da média total de acertos. O tempo foi outro fator positivo, onde a média de tempo foi de 14 minutos e 06 segundos para realizar todo o teste, diminuindo 3 minutos e 08 segundos na média final.

Tabela 2. Informação do Teste de Lógica 1

\begin{tabular}{|l|l|l|l|l|}
\hline \multicolumn{5}{|l|}{ Teste de Lógica 1 } \\
\hline & Questão 1 & Questão 2 & Questão 3 & Questão 4 \\
\hline Tempo Médio por questão & 2 min e $07 \mathrm{seg}$ & 1 min e $52 \mathrm{seg}$ & 9 min e $30 \mathrm{seg}$ & 3 min e $45 \mathrm{seg}$ \\
\hline Porcentagem por questão & $62,50 \%$ & $37,50 \%$ & $50,00 \%$ & $75,00 \%$ \\
\hline
\end{tabular}


No primeiro teste, é possível observar que levaram mais tempo na questão de tentativa e erro (Questão 3), em média de 9 minutos na questão, conforme a Tabela 2. A segunda questão com maior tempo dedicado foi a que envolvia imagens; as questões que envolviam apenas lógica, foram as que obtiveram mais facilidade em ser realizadas pois demandaram menor tempo para resolução.

Tabela 3. Informação do Teste de Lógica 2

\begin{tabular}{|c|c|c|c|c|}
\hline \multicolumn{5}{|l|}{ Teste de Lógica 2} \\
\hline & Questão 1 & Questão 2 & Questão 3 & Questão 4 \\
\hline Tempo Médio por questão & $3 \mathrm{~min}$ e $37 \mathrm{seg}$ & $3 \mathrm{~min}$ e $22 \mathrm{seg}$ & $2 \mathrm{~min}$ e $52 \mathrm{seg}$ & $4 \mathrm{~min}$ e $15 \mathrm{seg}$ \\
\hline Porcentagem por questão & $50 \%$ & $87,50 \%$ & $62,50 \%$ & $12,50 \%$ \\
\hline
\end{tabular}

No segundo teste, que foi aplicado no final do curso, foi observado um nivelamento em diversos fatores. Ao concluir o curso, os alunos desenvolveram ainda mais a sua lógica, logo, pensaram mais para fazer as questões de lógica, porque vão considerar mais possibilidades para resolver o problema. Sendo assim, levaram mais tempo nas questões de lógica do que as demais questões de lógica do primeiro teste (questões de tentativa e erro ou que demanda atenção, como identificação de padrões em imagens), como pode ser observado na Tabela 3.

\section{CONCLUSÃO}

Trabalhar com introdução a robótica e o ensino da lógica de programação com os alunos, de uma forma que fosse mais atrativa e de fácil interação, foi um desafio inicial no planejamento do projeto. Desafio esse que logo foi solucionado com a opção do uso do kit educativo LEGO® Mindstorms. Essa escolha se justificou pelo fato de o kit atender as necessidades da nossa ideia, isto é, apresentar uma interface visualmente atrativa e de fácil interação para quem não está familiarizado com a sequência de passos de uma programação. Além de permitir que a combinação dos comandos inseridos no software seja visualizada em ações no mundo real, como, o movimento dos motores dos robôs. Permitir aos alunos ver os seus códigos funcionarem no mundo físico e não somente no virtual pode tornar mais empolgante o aprendizado da lógica de programação.

Dos benefícios da assimilação de todo esse conhecimento por parte dos alunos, é possível citar a melhor adaptação dos mesmos no contexto da tecnologia hoje, podendo vir a despertar o desejo de futuramente ingressarem em algum curso na área das Ciências Computacionais.

Concluímos que a aplicação do curso, foi de grande aproveito para os alunos do ensino básico como para os integrantes do grupo que encontraram várias dificuldades durante a etapa de planejamento e realização do projeto. Podemos também concluir, a partir das médias totais de acerto, que no primeiro teste as notas eram muito dispersas, conforme o desvio padrão apontava. Já no segundo teste, as notas foram melhores, e quem havia se saído mal no primeiro teste, se saiu melhor no segundo, com isso o desvio padrão diminuiu, nivelando o conhecimento de todos. O que pretendíamos levar aos alunos, além, da lógica de programação propriamente dita, era instigá-los em uma nova maneira de pensar, de forma que eles possuíssem os conhecimentos necessários, ainda que de forma inicial. A curto prazo, a lógica de programação e a robótica podem vir a auxiliar esses alunos em avaliações que exijam pensamento lógico e abstrato. A longo prazo, esse conhecimento pode ser útil para o ingresso em algum curso de graduação na área das ciências exatas e/ou Engenharia. Os resultados foram muito satisfatórios e a experiência em trabalhar no ensino básico muito recompensadora, por esse motivo o projeto já se encontra na segunda edição e sendo realizado em duas escolas distintas. 
CBIE-LACLO 2015

Anais dos Workshops do IV Congresso Brasileiro de Informática na Educação (CBIE 2015)

\section{REFERÊNCIAS BIBLIOGRÁFICAS}

CARBONE, Felipe J.; SASS, Gláucia G. Ensinando programação e lógica computacional para a Olimpíada Brasileira de Informática em Dourados. Disponível em : Acesso em:5 jun. 2014.

DE OLIVEIRA, Gustavo B.; PEDRINI, Renata; MEIRINHO, Christian J.; BERGER, Felipe; WATANABE, Ana T. Y.; LEAL, André B.; Kits Lego® Mindstorms ${ }^{\circledR}$ NXT como ferramenta de interação entre Engenharia e Ensino Médio. In: XLI COBENGE, 2013, Gramado - RS.

MITCHELL, Matthew; SHEARD, Judy; MARKHAM, Selby. Student motivation and positive impressions of computing subjects. In: Proceedings of the Australasian conference on Computing education. ACM, 2000. p. 189-194.

ROGERS, Chris; PORTSMORE, Merredith; Bringing Engineering to Elementary School. In: Journal of STEM Education. Vol. 5, Issue 3 and 4, pp 17-28, 2014. 